\title{
Effect of exposure and withdrawal of pyethroid and herbal based mosquito vaporizers on histology of spinal cord of male wistar albino rats
}

\author{
Arvind Kumar Pankaj ${ }^{1}$, Manisha Pandey ${ }^{2, *}$, Punita Manik ${ }^{3}$, Anita Rani ${ }^{4}$, Sushma Tomar $^{5}$ \\ ${ }^{1}$ Associate Professor, ${ }^{2}$ Post Graduate, ${ }^{3,4}$ Professor, ${ }^{5}$ Assistant Professor, ${ }^{1-5}$ Dept. of Anatomy, ${ }^{1-5}$ King George's Medical University, \\ Lucknow, Uttar Pradesh, India \\ *Corresponding Author: Manisha Pandey \\ Email: drarvindpankajcsmmu@yahoo.com
}

\begin{abstract}
Introduction: Pyrethroids, organochlorines, organophosphates, and carbamates constitute important active ingredients of available mosquito repellents in the Indian market, in which pyrethroids are found very effective and safe. The natural compounds produced by plants can inter fere major metabolic pathways, thus causing death of the insects. The aim of present study was to know the effect of exposure and withdrawal of transfluthrin and herbal based mosquito vaporizers on spinal cord of male albino rats.

Materials and Methods: We have taken spinal cord of 26 well preserved male albino wistar rats and divided in to three groups.

Control Group: Six rats, notexposed to any mosquito repellents.

Experimental Group 1: 12 rats: divided in subgroup 1A, the pyrethroid Exposure group; and subgroup1B, the Pyrethroid withdrawal group.

Experimental Group 2: Eight rats: divided in subgroup 2A, the herbal Exposure group and 2B, the herbal withdrawal group.

Results: Spinal cord of pyrethroid vaporizer exposed group showed gross destruction in their histology than the herbal based mosquito vaporizer exposure group. Also, some of the normal histological features were recovered after withdrawal of chemicals and reversal was better in herbal based mosquito vaporizer.

Conclusion: Histological findings of present study suggests that pyrethroid (Transfluthrin) based mosquito repellents are harmful to the spinal cord. Herbal based mosquito repellent also showed damage though the severity was mild as compared to pyrethroid. Reversal changes were much better in herbal withdrawal group than the pyrethroid withdrawal group.
\end{abstract}

Keywords: Pyrethroid, Transfluthrin, Herbal, Mosquito repellent, Spinal cord, Withdrawal.

\section{Introduction}

In the race of better quality of life, knowingly or unknowingly we are exposing ourselves to many harmful chemicals in our daily routine, and protecting ourselves from mosquito bites is one such example. Mosquito borne diseases are burden on community health. There are many chemical mosquito repellents in the form of Mosquito Coils, Liquid Vaporizers are available in the market as personal protective measures from mosquito bite. Pyrethroids, organochlorines, organophosphates, and carbamates constitute important active ingredients of available mosquito repellents in the Indian market, in which pyrethroids are found very effective and safe. ${ }^{1}$

There are two categories of pyrethroids. Type I pyrethroids (like allethrin, resmethrin, and permethrin) does not contain an alfacyano group, while type II pyrethroid (like deltamethrin, cypermethrin, and fenvalerate) contains an alfacyano group. Type I usually causes T-syndrome characterized by tremors, whereas type II pyrethroids usually present its adverse effect in the form of CSsyndrome. ${ }^{2}$ The type I pyrethroid transfluthrin (TFL) is synthesized by tetrafluorobenzyl alcohol and trans-2,2dimethyl- 3-(2,2-dichlorovinyl) cyclopropanecarboxylate. ${ }^{3}$ Since plant produces a large number of secondary compounds like alkaloids, terpenoids, phenolics, flavonoids, chromenes which possess physiological activities against insectsso this may provide a new source of natural insecticides. These natural compounds produced by plants can interfere major metabolic pathways, thus causing death of the insects. ${ }^{4}$
There is a lack of studies on effect of herbal based mosquito vaporizers on histology of organs so the aim of present study was to know the effect of exposure and withdrawal of trans fluthrin and herbal based mosquito vaporizers on spinal cord of male albino rats.

\section{Material and Methods}

The present study was conducted in the Department of Anatomy, King George's Medical University, UP, Lucknow during August 2016 to September 2017. Ethical clearance was obtained from Institutional Animal Ethics committee (IAEC), King George's Medical University. The proposed study was carried out on various formalin fixed viscera from 26 male wistar albino rats.

\section{Inclusion Criteria}

Spinal cord from well preserved male wistar albino rats exposed to pyrethroid and herbal based mosquito vaporizers were taken for study.

\section{Exclusion Criteria}

Spinal cord of male albino wistar rats not well preserved with distorted morphology were excluded from the present study.

\section{Study Design}

Well-preserved spinal cord of 26 Male albino rats with, were divided into 3 groups: 


\section{Control Group}

Spinal cord of 6 male albino rats, those were not exposed to mosquito vaporizer fumes.

\section{Experimental Group I}

Spinal cord of 12 male albino rats (divided in subgroup I A and subgroup I B; each subgroup containsspinal cord of 6 male albino rats).

\section{Subgroup I A}

Contains spinal cord of male albino rats exposed to chemical-based mosquito vaporizer (Transfluthrin $0.88 \%$ w/w) for 8 hours /day, 6 days in a week for 12 weeks.

\section{Subgroup I B}

Contains spinal cord of male albino rats those were given exposure of chemical-based mosquito vaporizer (Transfluthrin $0.88 \% \mathrm{w} / \mathrm{w}$ ) for 8 hours/day, 6 days in a week for 12 weeks and further kept for 4 weeks to see the reversal changes.

\section{Experimental Group II}

Spinal cord of 8 male albino rats (divided in subgroup II A and subgroup II B; each subgroup contains organs of 4 male albino rats).

\section{Subgroup II A}

Spinal cord of male albino rats exposed to herbal based mosquito vaporizer for 8 hours/day, 6 days in a week for 12 weeks.

\section{Subgroup II B}

Contains spinal cord of male albino rats those were exposed to herbal based mosquito vaporizer for 8 hours/day, 6 days in a week for 12 weeks and further kept for 4 weeks to see the reversal changes.

\section{Products detail \\ Chemical Based Mosquito Vaporizer}

Good Knight Refill (Vaporizer containing 0.88\% transfluthrin, Reg. No. CIR-48,420/2004; manufactured by Godrej Consumer Products Ltd.) and herbal based Mosquito Vaporizer Mosrelief Refill Vaporizer (Vaporizer containing Cymbopogon citrius*, Bhutika, oil:6\%, Azardirachtaindica**, Nimbaoil:6\%, Eucalyptus Globulus, Tailaparna, oil:6\%, Exciepients: OS, *API, **AFI; Mff. Licence No. AUS920; manufactured by Strategi, Plot No.:50, Part 1, $4^{\text {th }}$ Phase, KIADB industrial area, Mular-563130, Kolardistt., Karnataka, India.) were used in our study.

Slides were prepared for histopathological observation, by routine Histological procedure i.e. fixation, clearing, paraffin embedding and sections of $5 \mu \mathrm{m}$ thick were cut by rotary microtome and stained with Hematoxylin and Eosin stain.

Light microscopic examination of sections of spinal cord was done to evaluate and compare the histopathological changes. Observation of slides was done under 10X, 40X, by Kyowa Trinocular Research Microscope. Microphotograph of different tissues were taken for documentation by digital camera (DSC-WX80/RC E32, manufactured in China by Sony corporation, 1-7-1 konan, Minto-KU, Tokyo 108-0075, Japan, month of import April 2014).

\section{Observations \\ Histological findings of spinal cord Control group}

At $10 \mathrm{X}$ magnification the histological section of spinal cord at cervical region showed intactciliated cuboidal ependymal linings of central canal. Both anterior and posterior grey horns were evident with their multipolar neurons. A large central nucleus, a prominent nucleolus, and several radiating cell processes were seen in the multipolar motor neurons of the spinal cord at higher magnification (40X). A single, thin axon was seen arising from a coneshaped, clear area of the neuron; axon hillock. Smaller cells with basophilic nuclei (neuroglia) were seen between spaces between the neurons (Fig. 1).

\section{Group IA}

Haematoxy line and Eosine stained section of spinal cord of pyrethroid exposed group showed discontinuity in ependymal linings of central canal (10X). There was extensive neuronal degeneration and perineuronal vacuolations of grey matter. Few neuroglial cells were seen (Fig.1.1). There was capillary dilatation and haemorrhage at some places in grey matter. The vacuolations of white matter was also seen at some places. The extensive oedema and loss of inter neuronal contacts leading to wide separation of grey and white matter was clearly visible (Fig.1.1).

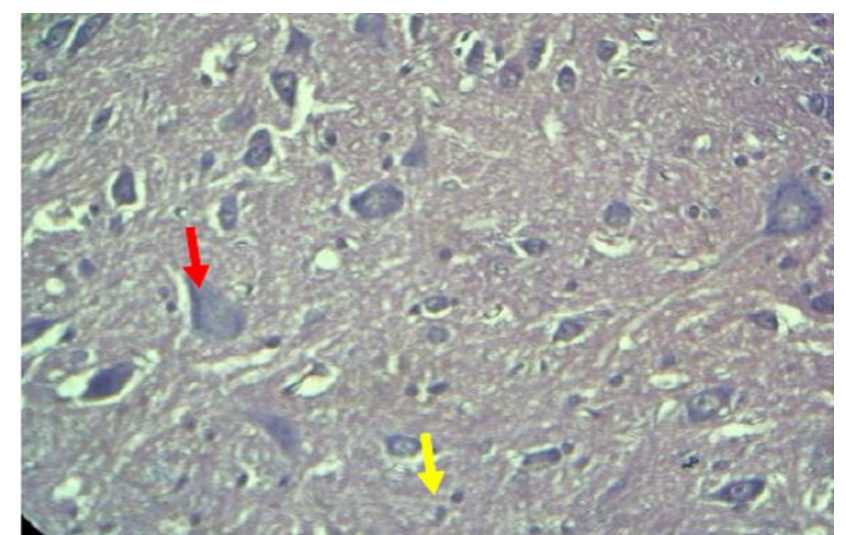

Fig. 1: (40X) Photograph of spinal cord of control group showing multipolar motor neuron (red arrow) and neuroglial cells (yellow arrow).

\section{Group IB}

The histological sections of group IB showed disruption of ependymal lining of central canal. Degeneration of neurons and perineuronal vacuolation of the grey matter was seen. There was loss of neuroglial cells (Fig.1.2) but it was less marked as compared to group 1A. Capillary dilatations 
and haemorrhage at some places in grey matter was observed. The vacuolations of white matter were less marked as compared to group IA. There was extensive oedema and loss of inter neuronal contacts resulted into separation of grey and white matter (Fig. 1.2).

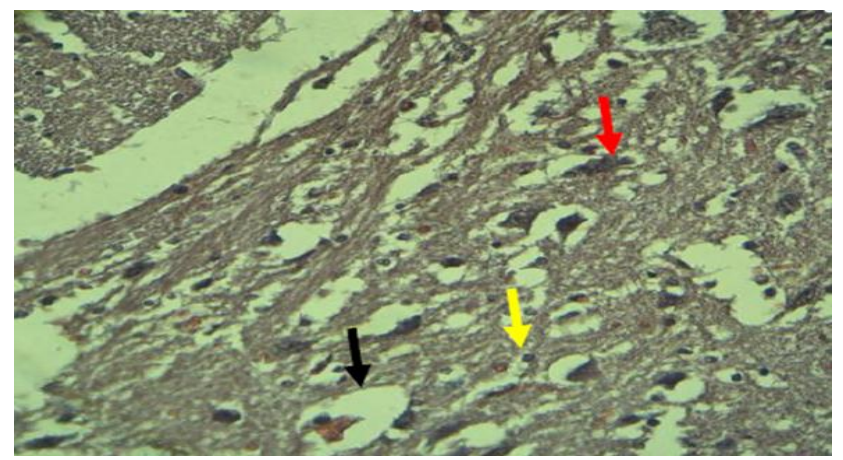

Fig. 1.1 (40X) Group IA showing neuronal degeneration (red arrow), perineuronal vacuolation (black arrow) and few neuroglial cells (yellow arrow).

\section{Group IIA}

Disruption of central canal lining and oedema was well evident in grey matter of spinal cord of Group IIA. Degeneration of neurons and loss of neuroglia of grey matter of spinal cord was also observed (Fig.1.3). There was sparsely placed axons and loss of neuroglia from the white matter (Fig.1.3).

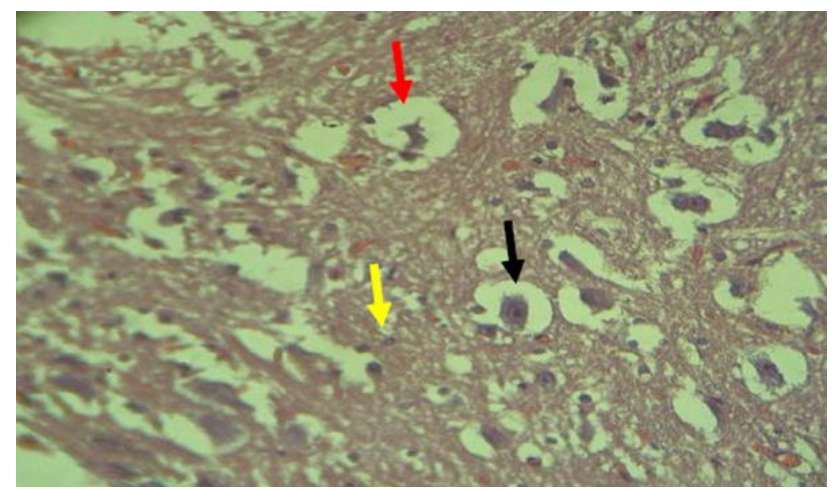

Fig. 1.2: (40X) Group IB showing neuronal degeneration (red arrow), perineuronal vacuolation (black arrow) and more neuroglial cells (yellow arrow).

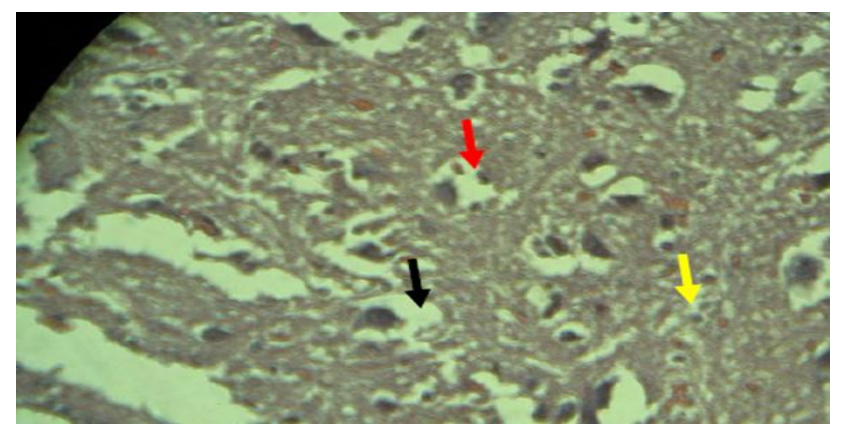

Fig. 1.3: (40X) Group IIA showing neuronal degeneration (red arrow), perineuronal vacuolation (black arrow) and few neuroglial cells (yellow arrow).

\section{Group IIB}

Intact central canal was clearly seen. Less oedematous grey matter. Most of the multipolar neurons showed axon hillock along with nissel's granules. There was densely packed nerve fibres and neuroglia almost similar to that of control group, were seen in the white matter (Fig.1.4).

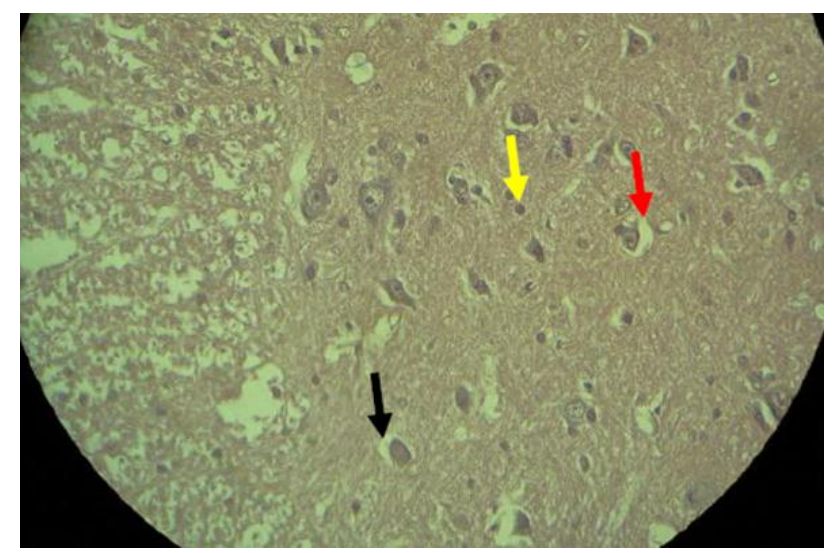

Fig. 1.4: (40X) Group IIB showing less marked neuronal degeneration (red arrow), perineuronal vacuolation (yellow arrow) and abundant neuroglial cells (black arrow).

\section{Discussion}

There are many insecticidal agents which cause variable changes in the central nervous system on repeated exposure. Metabolic changes due to hypoxia and hypoglycaemia can lead to cellular injury into the exposed organs.

H\&E stained section of spinal cord of pyrethroid exposure group of present study showed extensive neuronal degeneration, perineuronal vacuolation in grey matter and vacuolation of the white matter along with capillary dilatation. There was wide separation of grey and white matter which results due to oedema and loss of interneuronal contacts. Our findings are in accordance to a study of where sub lethal toxicity of cypermethrin in Sprague Dawley rats showed spongiosis and perineuronal degeneration of spinal cord and gliosis, degeneration of neurons and perineuronal vacuolation was also evident in the in the cerebrum of exposure group of rats. ${ }^{5}$ In another study, the rats, those were given orally the sub-lethal doses of pyrethroids showed various histopathological changes in peripheral nerves and spinal cord. ${ }^{6}$ Necrosis and degeneration of neurons of cerebrum was also reported among cypermethrin-intoxicated rats. ${ }^{7}$ Rats those were exposed to cypermethrin showed cellular injury in the form of pyknosis and loss of some cells in the cerebellum. ${ }^{8,9}$ Degeneration and congestive changes were reported in a study which was similar to the present observations. ${ }^{10,11,12}$ Concordant to our findings, haemorrhages were also observed in the brain tissue of rats exposed to cypermethrin. ${ }^{12}$ 


\section{Conclusion}

After evaluation of histological changes, it was seen that Inhalation of pyrethroid and herbal mosquito vaporizers both causes neuronal degeneration and spongiosis in spinal cord. Discontinuation of herbal vaporizer exposure for one month exhibits significant reversal of injury in comparison to withdrawal of pyrethroid based mosquito vaporizer. It can be concluded that toxic effects of these chemicals, whether synthetic or natural, are seen in rats. Therefore, we should limit the use of these vaporizers and further studies should be done to identify other compounds and its relatedtoxi city must be explored as a safer alternative for protection against these vectors.

\section{Conflicts of Interest: None.}

\section{References}

1. Zofou D, Nyasa RB, Nsagha DS, Ntie-Kang F, Meriki HD, Assob JCN, et al. Control of malaria and other vector-borne protozoan diseases in the tropics: enduring challenges despite considerable progress and achievements. Infect Dis poverty 2014;3(1):1.

http://www.pubmedcentral.nih.gov/articlerender.fcgi?artid=38 95778\&tool=pmcentrez\&rendertype $=$ abstract

2. Tordoir WF, Maroni M, He F. Health surveillance of pesticide workers. A manual for occupational health professionals (ICOH-ICPS-WHO, vol. 91). 1994

3. Pollack RJ, Kiszewski A, Armstrong P, Hahn C, Wolfe N, Rahman HA et al. Differential permethrin susceptibility of head lice sampled in the United States and Borneo. Arch Pediatr Adolesct Med 1999;153(9):969-973.

4. Bell, A. E.; Fellows, L. E. and Simmonds, S. J. Natural products from plants for the control of insect pests. In: E.
Hdgson and R. J. Kuhr, eds., Safer insecticides development and use morcelBekker, U.S.A. 1990.

5. Nair R, Abraham M, Lalithakunjamma C, Nair Nd, Aravindakshan C. A pathomorphological study of the sublethal toxicity of cypermethrin in Sprague Dawley rats. Int $j$ Nutr, Pharmacol, Neurol Dis 2011;1(2):179.

6. Vijverberg H, vandenBercken J. Neurotoxicological Effects and the Mode of Action of Pyrethroid Insecticides. Crit Rev Toxicol 1990;21(2):105-126.

7. Grewal KK, Sandhu GS, Kaur R, Brar RS, Sandhu HS; Toxic impacts of cypermethrin on behavior and histology of certain tissues of albino rats. Toxicol Int 2010;17(2):94-98.

8. Luty S, Latuszynska J, Halliop J, Tochman A, ObuChowska D, Prazylepa E et al. Toxicity of dermally applied alpha cypermethrin in rats. Ann Agric Environ Med 1998;5:109-15.

9. Latuszynska J, Luty S, Raszewski G, Roda MT, Przebirowska D, Przylepa E et al. Neurotoxic effects of dermally applied chlorpyriphos and cypermethrin in Wistar rats. Ann Agric Environ Med 2001;8:163-170.

10. Patel BJ, Singh SP, Sharma SN, Joshi DV. Clinicopathomorphological studies on induced cypermethrin toxicity in crossbred cow calves. Indian J Anim Sci 2000;70:925-928.

11. Muthuviveganandavel V, Muthuraman P, Muthu S, Srikumar K. A study on low dose cypermethrin induced histopathology, lipid peroxidation and marker enzyme changes in male rat. Pestic Biochem Physiol 2008;91(1):12-16.

12. Manna S, Bhattacharya D, Basak DK, Mandal TK. Single oral dose toxicity study of á cypermethrin in rats. Indian $J$ Pharmacol 2004;36:258

How to cite this article: Pankaj AK, Pandey M, Manik P, Rani A, Tomar S, Effect of exposure and withdrawal of pyethroid and herbal based mosquito vaporizers on histology of spinal cord of male wistar albino rats. Indian $J$ Anat Surg Head Neck Brain 2018;5(1):13-16. 